\title{
Non-Resonance Searches with Lepton
}

\author{
DongHee $\mathrm{Kim}^{1, \mathrm{a}}$ on behalf of ATLAS and CMS collaboration \\ ${ }^{1}$ WCU High Energy Collider Physics Research, Kyungpook National University, Daegu, 702-701, Republic of Korea
}

\begin{abstract}
In this contribution, results on non-resonance search with lepton are presented. The searches looking for a single lepton, dilepton, trileptons and quarks as well have been performed and their possible interpretations for each channel are given. The discussion is limited to very recent results presented by ATLAS and CMS collaboration in this symposium.
\end{abstract}

\section{Introduction}

Non-resonance searches are a typical example that the LHC took over quickly the all the recent results against the Tevatron due to the beam energy gaining an order of magnitude in resolution power for searches. The decay channels considered are a single lepton, dilepton and trileptons as well as quarks. The results, which are the most recent, here are based on either $7 \mathrm{TeV}$ or $8 \mathrm{TeV}$ data collected by the ATLAS and the CMS.

Numerous interpretations are possible to understand the each decay. Results from the single lepton decay could be interpreted as searches of charged heavy gauge boson from the Sequential Standard Model (SSM) [1], excited chiral bosons [2], Kaluza-Klein (KK) bosons inspired by split-Universal Extra Dimensions (UED) [3] and preon binding energy of Contact Interaction (CI) models [4]. Dilepton decay could also be interpreted with Large Extra Dimensions (LED) [5] and CI. Trileptons channel is rather background free to make easier searching for new phenomena expected from extended gauge models [1] and technicolor models [6][7]. The channels with quarks could be interpreted as searches of Heavy neutrino in left-right symmetric extension to the SM [8] and complementary new gauge bosons as well.

\section{A Single Lepton Signatures}

A single lepton channel in hadron collisions has been investigated for last few decades due to simplicities of decay mode. Mainly it has been searched for a new heavy gauge boson $W^{\prime}$ decaying to an electron or muon plus a low mass neutrino. However there are some additional interpretations considered by both the ATLAS and the CMS.

A search for exotic particles decaying to an electron or muon, plus a low mass neutrino, has been performed using 2012 data corresponding to an integrated luminosity of $3.7 \mathrm{fb}^{-1}$, collected using the CMS detector in pp collisions at a centre-of-mass energy of $8 \mathrm{TeV}$ at the LHC.

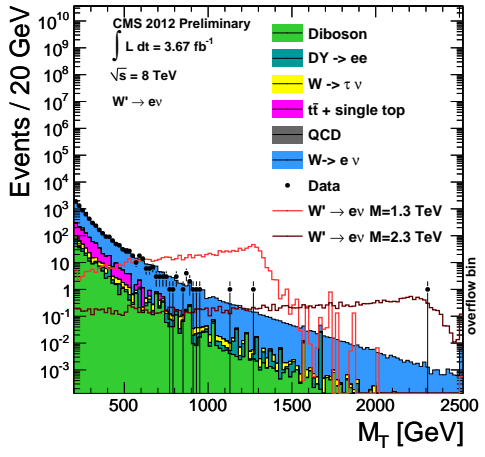

Figure 1. Observed transverse mass distribution for the $W^{\prime} \rightarrow$ ev channel. Simulated $W^{\prime}$ signal distributions are also shown, including detector resolution effects. Labeled 'dibson' includes $W W, Z Z$ and $W Z$ contributions.

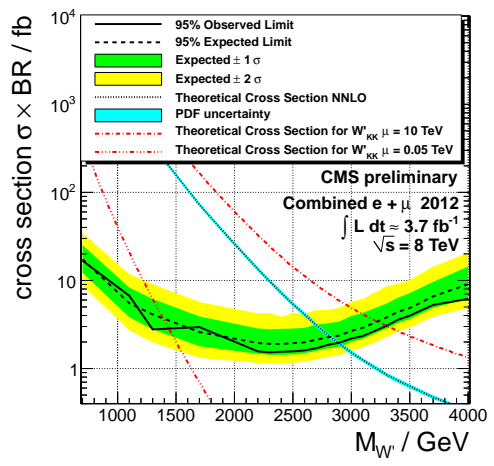

Figure 2. The limit curve with the combination of electron and muon channel of $W^{\prime}$ with two UED cross sections $(\mu=0.05$ and $10 \mathrm{TeV}$ ) plotted. The limits are derived with a Bayesian method.

a e-mail: dkim@knu.ac.kr 


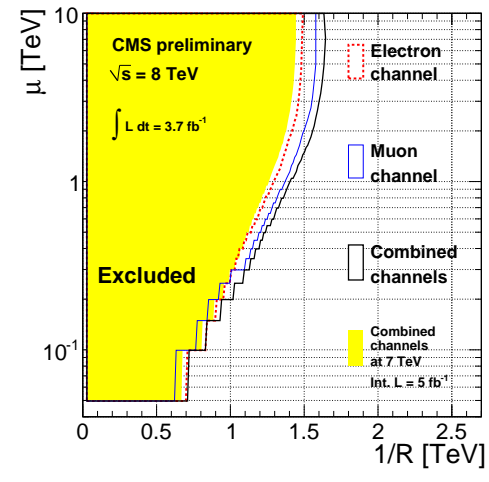

Figure 3. $95 \%$ C.L. limits on the split-UED parameters $\mu$ and $1 / \mathrm{R}$ derived from the $W^{\prime}$ mass limits taking into account the corresponding width of the $W_{\mathrm{KK}}^{2}$. The limit for the electron (red dotted) and the muon (blue) channel individually along with their combination in 2012 data is compared to the 2011 data combined result (yellow). The $W_{\mathrm{KK}}^{2}$ is the lowest state that can couple to SM fermions and has the same final state as the SM-like $W^{\prime}$.

Candidate events with at least one high $p_{\mathrm{T}}$ lepton were selected using single-muon (with $p_{\mathrm{T}}>40 \mathrm{GeV}$ ) and singleelectron (with $p_{\mathrm{T}}>85 \mathrm{GeV}$ ) triggers. Electrons were reconstructed as isolated objects in the electromagnetic calorimeter, with additional requirements on the shower shape and the ratio of hadronic to electromagnetic deposited energies. The electrons were required to have at least one inner tracker hit, a transverse energy greater than $90 \mathrm{GeV}$, and were required to be isolated in a cone of radius $\Delta \mathrm{R}=\sqrt{\Delta \eta^{2}+\Delta \phi^{2}}<0.3$ around the electron candidate direction, both in the tracker and in the calorimeter.The main observable in this search is the transverse mass $M_{\mathrm{T}}$ of the lepton-missing $E_{\mathrm{T}}$ system, calculated as $M_{\mathrm{T}}=\sqrt{2 \cdot p_{\mathrm{T}}^{l} \cdot E_{\mathrm{T}}^{\text {miss }} \cdot\left(1-\cos \Delta \phi_{l, v}\right)}$ where $\Delta \phi_{l, v}$ is the azimuthal opening angle between the charged lepton's transverse momentum and missing $E_{\mathrm{T}}$ direction. The transverse mass distributions [9] for $e v$ channel after these selections are shown in Figure 1.

The primary source of background for all these signals is the off-peak, high transverse mass tail of the standard model $W \rightarrow l v$ decays. Other important backgrounds arise from QCD multijet, $t \bar{t}$, Drell-Yan (DY) and Di-bosons $(W W, W Z, Z Z)$ events. The event samples for each background were generated by taking into account of NNLO cross sections mostly. All the samples were normalized to the integrated luminosity of the recorded data. All the backgrounds estimates are shown in Figure 1 together with signal Monte Carlo (MC) events.

No significant excess of events above the standard model expectation is found in the transverse mass distribution of the lepton-neutrino system. Mass exclusion limits at 95\% C.L. for plausible exotic particles are determined. Cross-section limits were derived using a Bayesian method with a uniform prior probability distribution for the signal cross section. Figure 2 displays the excluded $W^{\prime}$ cross section times branching ratio as a function of the $W^{\prime}$ mass. The combination of electron and muon chan-

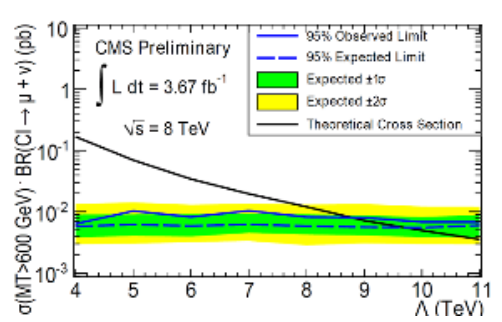

Figure 4. Limit on the preon binding energy $\Lambda$ in the helicitynon-conserving CI model ( $\Lambda<8.7 \mathrm{TeV}$ excluded)

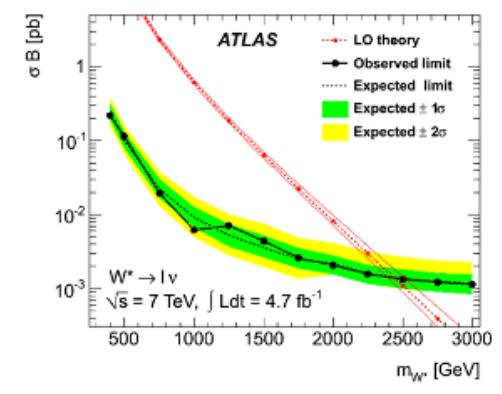

Figure 5. Expected and observed limits on $\sigma \times \mathrm{B}$ for $W^{*} \rightarrow l v$ in the combination of electron and muon channels assuming the same branching fraction for both. The calculated value for $\sigma \times$ $\mathrm{B}(\mathrm{LO})$ and its uncertainty is also shown.

nels for 2011 and 2012 data yields a limit on a SSM $W^{\prime}$ of $2.85 \mathrm{TeV}$. The observed limits illustrated in Figure 2 can also be reinterpreted in terms of the $W_{\mathrm{KK}}^{2}$ mass, as shown in the same figure for values of the bulk mass parameters $\mu=0.05$ and $10 \mathrm{TeV}$. These lower limits on the mass can be directly translated to bounds on the split-UED [10] parameter space $[1 / \mathrm{R}, \mu]$ as shown in Figure 3. Another reinterpretation can be done in terms of four-fermion CI of Helicity-Non-Conserving model [11], providing a limit on the preon binding energy scale $\Lambda$. The limit on $\Lambda$ is calculated to be $8.7 \mathrm{TeV}$ as shown in Figure 4. A search by ATLAS was performed for the charged partners [2][12], $W^{*}$, of the chiral boson excitations. The anomalous coupling of the $W^{*}$ leads to kinematic distributions significantly different from those of the $W^{\prime}$. A lower mass limit is evaluated by fixing the $W^{*}$ coupling strengths to give the same partial decay widths as the SSM $W^{\prime}$. A $W^{*}$ is excluded for $m_{W^{*}}<$ $2.42 \mathrm{TeV}$ [13] at the 95\% C.L. as shown in Figure 5.

\section{Dilepton Signatures}

Non-resonant deviations in the high mass dilepton invariant mass spectra are predicted in LED and CI. Both the ATLAS and the CMS has carried out the searches from the $e e$ and $\mu \mu$ channels. The left-left isoscalar model [14] is used as a benchmark for CI searches to set the limit of the scale $\Lambda$ while the three different formalisms, GRW [15], Hewett [16] and HLZ [17] in the ADD large extra dimensions model [5] to set the $M_{S}$ bound. 


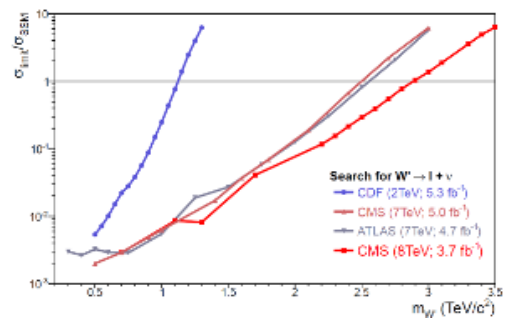

Figure 6. Evolution of $W^{\prime}(\rightarrow l v)$ mass limits for the last two years. Normalised cross-section limits $\left(\sigma_{\text {limit }} / \sigma_{\mathrm{SM}}\right)$ as a function of $W^{\prime}$ mass from CDF [36], ATLAS [13] and CMS [9][37]. The region above each curve is excluded at the $95 \%$ C.L.

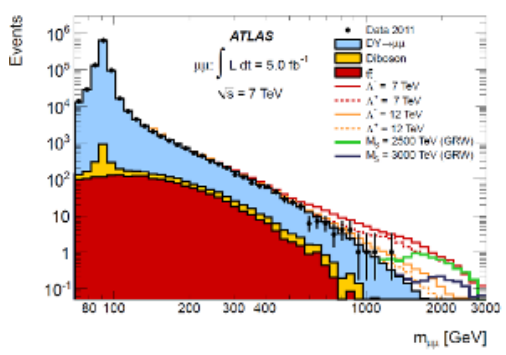

Figure 7. Dimuon invariant mass distribution for data (points) and MC simulation (filled histograms). The open histograms correspond to the distributions expected in the presence of CI or LED for several model parameters.

The ATLAS data sample corresponds to a total integrated luminosity of 4.9 and $5.0 \mathrm{fb}^{-1}$ at $7 \mathrm{TeV} p p$ collisions in the $e e$ and $\mu \mu$ final states, respectively. The trigger for the dielectron dataset required the presence of two electromagnetic clusters consistent with originating from electrons with transverse momentum $p_{\mathrm{T}}>20 \mathrm{GeV}$, whereas events in the muon dataset were required to pass at least one of two single-muon triggers with $p_{\mathrm{T}}$ thresholds of $22 \mathrm{GeV}$ and $40 \mathrm{GeV}$. After passing the trigger selection, events are required to have a pair of either electrons or muons with $p_{\mathrm{T}}>25 \mathrm{GeV}$. To reject cosmic ray events and beam halo background, events are required to have a reconstructed vertex with at least three charged particle tracks with $p_{\mathrm{T}}>0.4 \mathrm{GeV}$. If more than one such vertex is found, the vertex with the largest $\Sigma p_{\mathrm{T}}^{2}$ is selected as the primary vertex of the event, where the sum is over all charged particles associated with the given vertex. Electron candidates are confined to $|\eta|<2.47$, with the calorimeter barrel-to-endcap transition region $1.37 \leq|\eta| \leq 1.52$ excluded due to the degraded energy resolution in this region. Muon candidates are required to be within the inner detector acceptance. The electron and muon candidate are reconstructed.

The dominant background contribution comes from the SM DY process with smaller contributions from $t \bar{t}$ and electroweak diboson production. In the dielectron channel, there is also a significant background from multi-jet and $W+$ jets events in which jets are misidentified as electrons. Backgrounds are estimated using fully simulated MC samples except for the combined multi-jet and $W+$ jets
Table 1. Expected and observed 95\% C.L. lower limits on the CI energy scale $\Lambda$ for the combination of the dielectron and dimuon channels by ATLAS. Results are provided for constructive and destructive interference as well as different choices of flat priors: $1 / \Lambda^{2}$ and $1 / \Lambda^{4}$.

\begin{tabular}{llllll}
\hline channel & prior & $\begin{array}{l}\text { Exptd } \\
\text { Constr. }\end{array}$ & $\begin{array}{l}\text { limit } \\
\text { Destr. }\end{array}$ & $\begin{array}{l}\text { Obsrvd } \\
\text { Constr. }\end{array}$ & $\begin{array}{l}\text { limit } \\
\text { Destr. }\end{array}$ \\
\hline$e e+\mu \mu$ & $1 / \Lambda^{2}$ & 15.0 & 11.3 & 13.9 & 10.2 \\
& $1 / \Lambda^{4}$ & 13.8 & 10.5 & 12.9 & 9.8 \\
\hline
\end{tabular}

Table 2. Observed 95\% C.L. lower limits on $M_{S}$ by ATLAS, including systematic uncertainties, for ADD signal in the GRW, Hewett and HLZ formalisms with K-factor applied to the signal for all the channels. Separate results are provided for the different choices of flat priors: $1 / M_{S}^{4}$ and $1 / M_{S}^{8}$.

\begin{tabular}{llllll}
\hline channel & prior & GRW & Hewett & \multicolumn{2}{l}{ HLZ $(\mathrm{n}=3, \mathrm{n}=7)$} \\
\hline$e e+\mu \mu$ & $1 / M_{S}^{4}$ & 3.51 & 3.14 & 4.18 & 2.79 \\
$+\gamma \gamma$ & $1 / M_{S}^{8}$ & 3.39 & 3.20 & 3.69 & 3.02 \\
\hline
\end{tabular}

background, which is determined from the data. Figure 7 shows dimuon invariant mass distribution [18] for data and MC simulation. The open histograms correspond to the distributions expected in the presence of contact interactions or large extra dimensions for several model parameters.

No significant deviation from the Standard Model is observed in the dilepton mass distributions. Using a Bayesian approach with a prior flat in $1 / \Lambda^{2}$, as was done in most previous searches at hadron colliders, the following 95\% C.L. limits are set on the energy scale of contact interactions: $\Lambda^{+}>13.9 \mathrm{TeV}\left(\Lambda^{-}>10.2 \mathrm{TeV}\right)$ in the combined dielectron and dimuon channel for constructive (destructive) interference in the left-left isoscalar compositeness model (Table 1). Somewhat weaker limits are obtained with a prior flat in $1 / \Lambda^{4}$. Limits are also set on the scale $M_{S}$ in the ADD model. Those range from 2.8 to $4.2 \mathrm{TeV}$ depending on the choice of model, channel, and prior for the combination of the dilepton and diphoton searches (Table 2). The limit by the CMS is $\Lambda^{+}>13.1 \mathrm{TeV}\left(\Lambda^{-}>9.5\right.$ $\mathrm{TeV}$ ) in the dimuon channel [19] for constructive (destructive) interference case with $5.3 \mathrm{fb}^{-1}$ at $7 \mathrm{TeV}$. For the $M_{S}$, depending on the number of extra dimensions and the validity range of the theory, limits of up to $3.72 \mathrm{TeV}$ at $95 \%$ C.L. were set in dielectron channels [20] with $5.3 \mathrm{fb}^{-1}$ at $7 \mathrm{TeV}$.

\section{Trilepton Signatures}

Even though $W^{\prime}$ searches have been conducted in leptonic final states by assuming that the $W^{\prime} \rightarrow W Z$ decay mode is suppressed, the searches by $W Z$ pairs are complementary to searches in the leptonic channels. Moreover, there are other models in which the $W^{\prime}$ couplings to SM fermions are suppressed, giving rise to a fermiophobic $W^{\prime}$ with an enhanced coupling to $W$ and $Z$ bosons [21][22]. As another interpretation of $W Z$ decay is technicolor modeling on QCD with no elementary scalar particles. In low-scale technicolor [23][24], the lightest techni-hadrons are expected to have masses below $700 \mathrm{GeV}$, with the lightest 


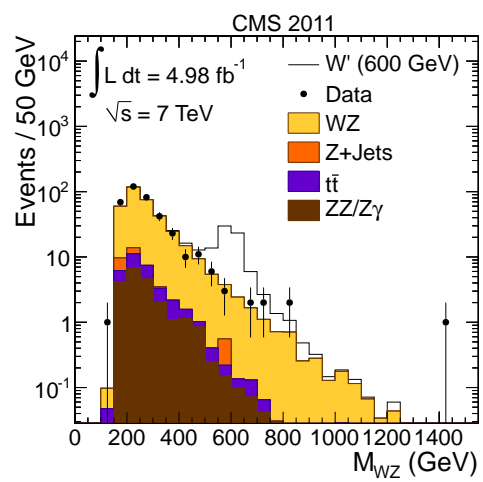

Figure 8. $W Z$ invariant mass distribution for the full mass range.

vector technirho $\rho_{\mathrm{TC}}$ and its axial-vector partner techni-a $a_{\mathrm{TC}}$ able to decay to $W Z$ boson pairs.

A search is performed in $p p$ collisions at $\sqrt{s}=7 \mathrm{TeV}$ for those particles decaying via $W Z$ to final states with electrons and muons by both the ATLAS and the CMS. The $W Z \rightarrow 3 l v$ decay is characterized by a pair of sameflavor, opposite-charge, isolated leptons with high transverse momentum, having an invariant mass consistent with that of the $Z$ boson, along with a third, high- $p_{\mathrm{T}}$, isolated lepton, and missing transverse energy associated with the escaping neutrino.

The dominant background for the $W Z$ search comes from SM $W Z$ production. Its contribution is estimated using MC simulation. Other sources of background, considered to be very small, are non-resonant events with no genuine $Z$ boson in the final state, including $t \bar{t}$, multijet, $W+$ jet, $W \gamma$, and $W W+$ jet production. Other diboson processes such as $Z Z$ and $Z \gamma$ are also estimated using MC simulation. Due to known mass of $W$ and $Z$ boson, both transverse mass and invariant mass distributions are possible. The ATLAS uses $M_{\mathrm{T}}$ distribution while the CMS adopts $W Z$ invariant mass distribution. The observed invariant mass distribution [25] of $W Z$ candidates by the CMS is shown in Figure 8. The mass distribution of the selected $W Z$ candidates is consistent with the SM expectation.

In the case of the ATLAS, using the mass hierarchy assumption $m_{\rho_{\mathrm{TC}}}=m_{\pi_{\mathrm{TC}}}+m_{W}, \rho_{\mathrm{TC}}$ technimesons with masses from $200 \mathrm{GeV}$ up to $467 \mathrm{GeV}$ and $456 \mathrm{GeV}$ are excluded at $95 \%$ C.L. for $m_{a_{\mathrm{TC}}}=1.1 m_{\rho_{\mathrm{TC}}}$ and $m_{a_{\mathrm{TC}}} \gg m_{\rho_{\mathrm{TC}}}$, respectively with $1.02 \mathrm{fb}^{-1}$ at $7 \mathrm{TeV}$ as shown in Figure 9 [26]. The CMS has set the most stringent limits to data in $W Z$ channel. Technicolor $\rho_{\mathrm{TC}}$ hadrons with masses between 167 and $687 \mathrm{GeV}$ have been excluded, assuming $m_{\rho_{\mathrm{TC}}}=m_{\pi_{\mathrm{TC}}}+25 \mathrm{GeV}$. Under the alternative assumption $m_{\rho_{\mathrm{TC}}}<m_{\pi_{\mathrm{TC}}}+m_{W}, \rho_{\mathrm{TC}}$ hadrons with masses between 180 and $938 \mathrm{GeV}$ have been excluded. Of particular anomalous event by the CDF in the $W+$ jets channel [27], with proposed parameters $m_{\rho_{\mathrm{TC}}}=290 \mathrm{GeV}$ and $m_{\pi_{\mathrm{TC}}}=160 \mathrm{GeV}$ is excluded by the $95 \%$ C.L. upper bound of $150 \mathrm{GeV}$ on $m_{\pi_{\mathrm{TC}}}$ for the required $\rho_{\mathrm{TC}}$ mass. $(\star$ point in Figure 10$)$

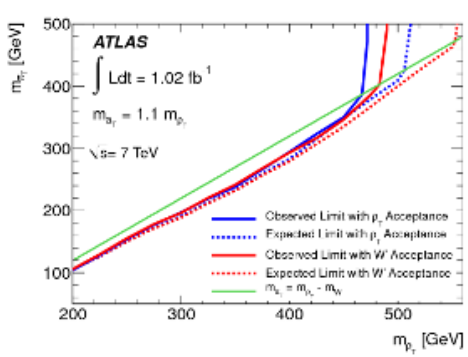

Figure 9. The 95\% C.L. expected and observed excluded mass regions in the $\left(m_{\rho_{\mathrm{TC}}}, m_{\pi_{\mathrm{TC}}}\right)$ plane for $m_{a_{\mathrm{TC}}}=1.1 m_{\rho_{\mathrm{TC}}}$.

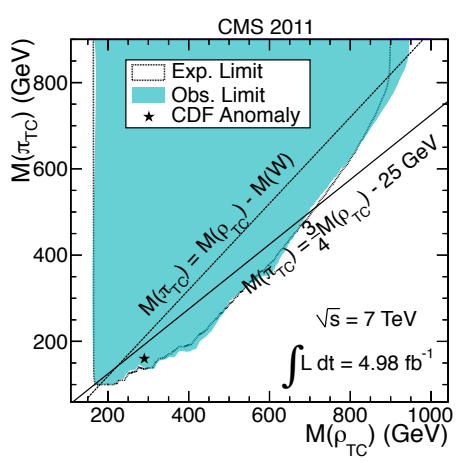

Figure 10. Two-dimensional exclusion limit for Technicolor as a function of the $\rho_{\mathrm{TC}}$ and $\pi_{\mathrm{TC}}$ masses. This plot uses $\sin \chi=1 / 3$.

\section{Signatures with quarks involved in}

A search for signals from the production of right-handed $W_{\mathrm{R}}$ bosons and heavy neutrinos $N$, that arise naturally in the left-right symmetric extension [28][29] to the SM, is performed by the CMS with $3.6 \mathrm{fb}^{-1}$ at $8 \mathrm{TeV}$. A heavy right-handed charged gauge boson $\left(W_{\mathrm{R}}\right)$ decays to a electron or muon and a heavy neutrino, $p p \rightarrow W_{\mathrm{R}} \rightarrow l N$, with the heavy neutrino subsequently decaying to a lepton and two jets via a virtual $W_{\mathrm{R}}$. The final state is thus two sameflavor leptons along with two jets. The primary backgrounds are top quark pair-production and DY production in association with jets. Multiboson, QCD multijet, and single top quark backgrounds contribute at a smaller rate.

The invariant mass distribution [30] of the electronelectron-jet-jet system for events surviving selection criteria is shown in Figure 11. The distribution of expected backgrounds and the signal mass point $M_{W_{\mathrm{R}}}=1800 \mathrm{GeV}$, $M_{N}=900 \mathrm{GeV}$, are included for comparison. No excess over expectations from Standard Model processes is observed. The exclusion of the region in the two-dimensional parameter $\left[M_{W_{\mathrm{R}}}, M_{N}\right]$ space extends beyond $M_{W_{\mathrm{R}}}=2.8$ $\mathrm{TeV}$ by combining the $8 \mathrm{TeV}$ electron and muon channel. Moreover the mass of $M_{W_{R}}$ was excluded up to $2.9 \mathrm{TeV}$ by combining 7 and $8 \mathrm{TeV}$ data for the muon channel only, and assuming that only the muon right-handed neutrino is light enough to be produced at the LHC as shown in Figure 12 .

As a different scenario [31][32], a heavy Majorana neutrino can be generated in association with a lepton 


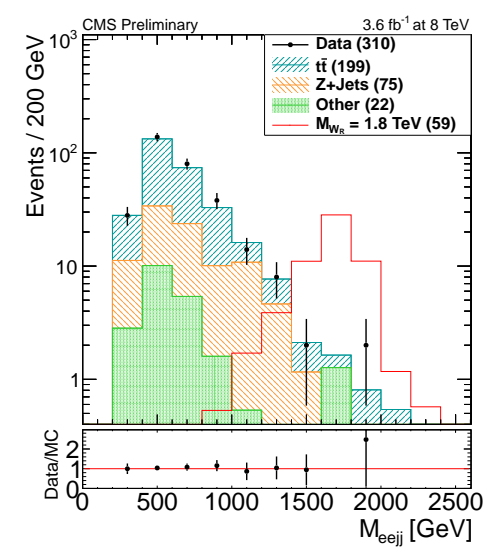

Figure 11. Distribution of the invariant mass of the eejj events by CMS. The distribution of expected backgrounds and the signal mass point $M_{W_{\mathrm{R}}}=1800 \mathrm{GeV}, M_{N}=900 \mathrm{GeV}$, are included for comparison.

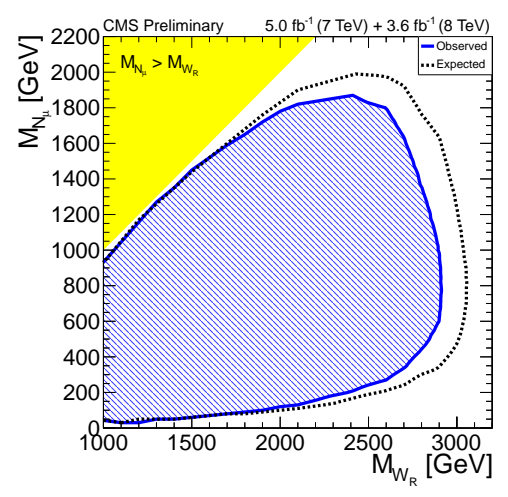

Figure 12. The $95 \%$ C.L. exclusion region for the muon channel, combining the $3.6 \mathrm{fb}^{-1}$ of $8 \mathrm{TeV}$ data with $5.0 \mathrm{fb}^{-1}$ of $7 \mathrm{TeV}$ data by CMS, as a function the mass of $W_{\mathrm{R}}$ and $N_{\mu}$, for the model assuming equal coupling in the left and right sectors of the model and with $v_{\mu}$ channel as the only available leptonic decay channel.

via $p p \rightarrow W^{ \pm} \rightarrow l^{ \pm} N$, with $N$ decaying subsequently to $N \rightarrow l^{ \pm} W^{\mp} \rightarrow l^{ \pm} j j$ where the $W$ produced from the $p p$ interaction is offshell. The search is performed by selecting events with two same-sign muons and at least two jets with low missing transverse momentum, using a dataset corresponding to an integrated luminosity of $4.7 \mathrm{fb}^{-1}$ at 7 $\mathrm{TeV}$ by the ATLAS. The SM background for this signature is small and comes from the production of diboson events, together with events where one or more of the muons originates from a hadronic decay.

The figure 13 shows the invariant mass of the two jets with the largest transverse momenta after the missing $E_{\mathrm{T}}$ selection is applied. A reasonable agreement is seen between the data and prediction from the background estimates indicating no excess of events observed. The $95 \%$ C.L. limits on the cross section times branching ratio was set. The cross section limits are translated into limits on the coupling parameter $\left|V_{\mu N}\right|^{2}$. The resulting limits [33] on the coupling are shown in Figure 14. These results are the most stringent direct limits to date on heavy Majorana neu-

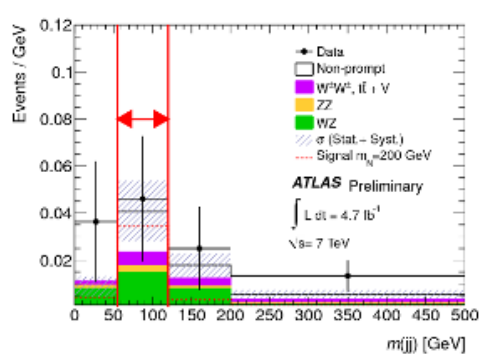

Figure 13. The invariant mass distribution of the two jets with the largest $p_{\mathrm{T}}$ for events with two same-sign muons, at least two jets and low missing transverse momentum. The shaded band shows the total uncertainty on the background prediction. The arrows indicate the regions retained by the selection requirements.

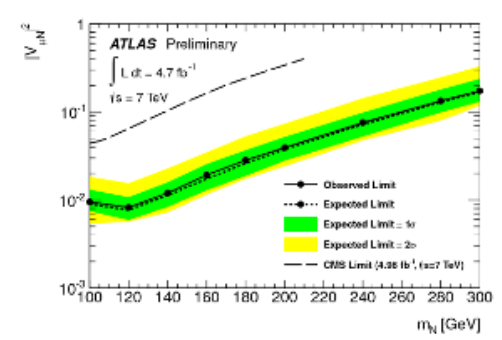

Figure 14. Observed and expected 95\% C.L. limits on the coupling parameter $\left|V_{\mu N}\right|^{2}$ as a function of the $M_{N}$ by the ATLAS. The observed limits from the CMS are also shown.

trino production for heavy neutrino masses greater than $100 \mathrm{GeV}$.

A search for $W_{R}^{\prime}$, that couples only to right-handed fermions [34], via the $W_{R}^{\prime} \rightarrow t b$ is complementary one another with the $l v$ and $W Z$ decays. The final state signature is an isolated lepton $(e$ or $\mu)$, an undetected neutrino and jets, at least one of which is tagged as a b-jet from the decay chain $W_{\mathrm{R}}^{\prime} \rightarrow t b, t \rightarrow b W \rightarrow b l v$.

No evidence for $W_{\mathrm{R}}^{\prime}$ boson production is found and 95\% C.L. upper limits on the production cross section times branching ratio [35] are set for arbitrary mixtures of couplings to left- and right-handed fermions. The measurement is compared to the theoretical prediction for the nominal value of the cross section to determine the lower limits on the mass of the $W_{\mathrm{R}}^{\prime}$. A limit of $1.85 \mathrm{TeV}$ is established as shown in Figure 15. In addition, constraints on the $W_{\mathrm{R}}^{\prime}$ gauge coupling for a set of left- and right-handed coupling combinations have been placed. The Figure 16 shows the contours for the $W_{\mathrm{R}}^{\prime}$ boson mass in the $\left(a^{\mathrm{L}}, a^{\mathrm{R}}\right)$ plane for which the cross-section limit equals the predicted cross-section. For each contour of $W_{\mathrm{R}}^{\prime}$ mass, combinations of $a^{\mathrm{L}}$ and $a^{\mathrm{R}}$ above to the right of the curve are excluded. These results represent a significant improvement over previously published limits.

\section{ACKNOWLEDGEMENTS}

This work was supported by the World Class University(WCU) program of the National Research Foundation of Korea (Grant R32-2008-000-20001-0). 


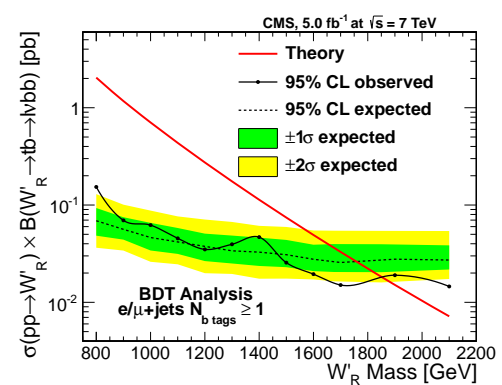

Figure 15. The expected and observed $95 \%$ C.L. upper limits on the production cross section $\sigma\left(p p \rightarrow W_{\mathrm{R}}^{\prime}\right) \times B\left(W_{\mathrm{R}}^{\prime} \rightarrow t b \rightarrow l v b b\right)$ of $W_{\mathrm{R}}^{\prime}$ bosons obtained using the BDT discriminant for $>1 b$ tagged $e+$ jets and $\mu+$ jets combined events. The solid red line represents the theoretical prediction.

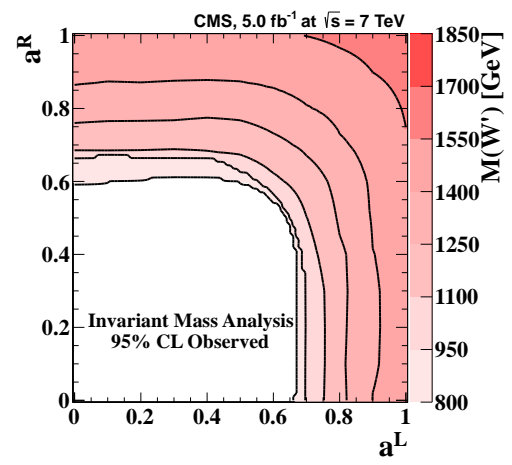

Figure 16. Contour plots of $W_{\mathrm{R}}^{\prime}$ in the $\left(a^{\mathrm{L}}, a^{\mathrm{R}}\right)$ plane at which the $95 \%$ C.L. upper cross section limit equals the predicted cross section for the combined $e, \mu+$ jets sample. The plot is the observed cross section limits. The color-scale axis shows the $W_{\mathrm{R}}^{\prime}$ mass in $\mathrm{GeV}$.

\section{References}

[1] G. Altarelli, B. Mele and M. Ruiz-Altaba, Z. Phys. C 45, 109 (1989)

[2] M. V. Chizhov, V. A. Bednyakov and J. A. Budagov, Phys. At. Nucl. 71, 2096 (2008)

[3] C.-R. Chen, M. M. Nojiri, S. C. Park et al, JHEP 09, 078 (2009)

[4] K. A. H. Terazawa, M. Yasue and M. Hayashi, Phys. Lett. B 112, 387 (1982)

[5] N. Arkani-Hamed, S. Dimopoulos, and G. Dvali, Phys.Lett. B 429, 263 (1998)

[6] S. Weinberg, Phys. Rev. D 19, 1277 (1979)

[7] L. Susskind, Phys. Rev. D 20, 2619 (1979)

[8] A. Ferrari, J. Collot, M.-L. Andrieux et al., Phys. Rev. D 62, 013001 (2000)

[9] CMS collaboration, https:// twiki.cern.ch /twiki /bin /view /CMSPublic /PhysicsResultsEXO12010 (2012)
[10] K. Kong, S. C. Park, and T. G. Rizzo, JHEP 04, 081 (2010)

[11] K. Lane, F. Paige, T. Skwarnicki et al, Phys. Rept. 278, 291 (1997)

[12] M. Chizhov, G. Dvali, Phys. Lett. B 703, 593 (2011)

[13] ATLAS collaboration, Eur. Phys. J. C 72, 2241 (2012)

[14] E. Eichten, I. Hinchliffe, K. D. Lane, and C. Quigg, Rev. Mod. Phys. 56, 579 (1984)

[15] G. F. Giudice, R. Rattazzi, and J. D. Wells, Nucl. Phys. B 544, 3 (1999)

[16] J. L. Hewett, Phys. Rev. Lett. 82, 4765 (1999)

[17] T. Han, J. D. Lykken, and R.-J. Zhang, Phys. Rev. D 59, 105006 (1999)

[18] ATLAS collaboration, Phys. Rev. D 87, 015010 (2013)

[19] CMS collaboration, accepted by Phys. Rev. D, arXiv: 1212.4563v1 [hep-ex] (2013)

[20] CMS collaboration, https:// twiki.cern.ch /twiki /bin /view /CMSPublic /PhysicsResultsEXO12013 (2012)

[21] K. Nakamura et al., J. Phys. G 37, 075021 (2010)

[22] H.-J. He, Y.-P. Kuang, Y.-H. Qi, B. Zhang, A. Belyaev, R. S. Chivukula, N. D. Christensen, A. Pukhov, and E. H. Simmons, Phys. Rev. D 78, 031701 (2008)

[23] E. Eichten and K. Lane, Phys. Lett. B 669, 235 (2008)

[24] S. Catterall, L. Del Debbio, J. Giedt, and L. Keegan, Phys. Rev. D 85, 094501 (2012)

[25] CMS collaboration, Phys. Rev. Lett. 109, 141801 (2012)

[26] ATLAS collaboration, Phys. Rev. D 85, 112012 (2012)

[27] CDF Collaboration, Phys. Rev. Lett. 106, 171801 (2011)

[28] J. C. Pati and A. Salam, Phys. Rev. D 10, 275 (1974)

[29] W.-Y. Keung and G. Senjanovic, Phys. Rev. Lett. 50, 1427 (1983)

[30] CMS collaboration, CMS-PAS-EXO-12-017 (2012) http: //cds.cern.ch /record /1460445

[31] T. Han and B. Zhang, Phys. Rev. Lett. 97, 171804 (2006)

[32] A. Atre, T. Han, S. Pascoli, and B. Zhang, JHEP 0905, 030 (2009)

[33] ATLAS collaboration, ATLAS-CONF-2012-139 (2012) http://cds.cern.ch/record/1480645

[34] E. H. Simmons, Phys. Rev. D 55, 5494 (1997)

[35] CMS collaboration, accepted by Phys. Lett. B, arXiv:1208.0956 [hep-ex] (2013)

[36] CDF collaboration, Phys. Rev. D 83, 031102 (2011)

[37] CMS collaboration, JHEP 08, 023 (2012) 